\title{
A conceptual approach to address the issue of hypertension in African Americans living in south west Philadelphia
}

\begin{abstract}
Cardiovascular diseases are the leading cause of death, globally. ${ }^{1}$ About 68 percent of the cardiovascular diseases are associated with hypertension in adults. Hypertension is asymptomatic in the early stage and shows symptoms only when it worsens. ${ }^{2}$ Therefore, it is essential to educate the patient about the disease's nature and the ill effects of untreated hypertension. According to statistics provided by national data, among the 10 largest counties in U.S where the prevalence of hypertension is common, adult hypertension is most common in Philadelphia. The community health assessment provided by the Philadelphia Department of Public Health (PDPH) states that the prevalence of hypertension is more common among African Americans residing in the Southwest Philadelphia. In addition to that it states that more than $37.5 \%$ of the adult residing in Philadelphia has been diagnosed with Hypertension. ${ }^{3}$ The main aim of the proposed project is to develop an intervention for treating, controlling and early detection of hypertension in African American's (AA) over the age of 40years in Southwest Philadelphia.
\end{abstract}

Keywords: hypertension, Philadelphia
Volume 2 Issue 3 - 2015

\section{Sneha Srinivasan}

Drexel University, USA

Correspondence: Sneha Srinivasan, Drexel University, 3605 Baring St APT 3R, Philadelphia, USA, Tel 8455193505,

Email sneha.srini09@gmail.com

Received: March 20, 2015 | Published: June 02, 2015

\section{Abbreviations}

PDPH, Philadelphia department of public health; AA, African American's; PPM, precede-proceed model; NHLBI, national heart, lung and blood institute; BP, blood pressure; CDC, centers for disease control and prevention; TJU, Thomas Jefferson university

\section{Introduction}

\section{The precede-proceed model}

The proposed intervention for this disease is constructed on the basis of the PRECEDE-PROCEED model (PPM). The PPM focuses on the community, behavior and health perspective of an intervention. The PPM provides a structured, systematic and in-depth framework for planning, implementing and evaluating the intervention which are arranged in detailed procedures. The involvement of the community as the major component of the intervention promotes greater community support and increases the chances of successful intervention. The PPM is comprised of the final outcome assessment and evaluation of the proposed project as a part of the intervention. It monitors the outcomes of the intervention and tailor's it according to the target population's needs. It guides the intervention to achieve its vision and goals. ${ }^{4}$ The proposed intervention for this concern is the TREATIT program which stands for test, reduce, educate, aware and treatintervention.

\section{Substantiation of the proposed approach}

AA's adults over 40years of age and those with low socioeconomic status are the most affected by hypertension (National Heart, Lung and Blood Institute [NHLBI]). AA's are more affected by hypertension than any other racial and ethnic groups. ${ }^{5}$ They constitute the target population of the intervention. Therefore, the intervention should focus on these root causes of the problem and should be shaped accordingly. The main aim of this intervention is to educate AA's in Southwest Philadelphia about the nature of the disease and the risk associated with untreated hypertension. Through education AA's can be encouraged to undergo regular blood pressure (BP) screening which will help them to maintain their BP levels. A study should be done for better understanding of this issue which will help in designing an education, awareness and screening program. Community gatherings and community-based programs should be conducted as a part of the intervention. The AA's in Southwest Philadelphia should be motivated and included in the designing of the intervention, implementation and evaluation. This will also encourage them to participate in the intervention program and eliminate their low confidence level and the inferiority complex. They will feel that they have been given importance and respect, which will motivate them to bring about a change in the community with respect to health. Therefore, involving the community leads to success of the intervention. ${ }^{4}$

\section{Conceptual approach}

The phase one of the PPM is the social assessment phase which focuses on defining the ultimate outcomes of the intervention. It asks the community and the AA's in Southwest Philadelphia what they want out of this intervention and what they feel about this problem. The information obtained will facilitate in modifying the intervention according to the needs of AA's. The assessment should be done with the help of community surveys, questionnaires and focus groups. The questionnaires and the survey forms will be printed in two languages, English and Spanish which will reduce the language barrier as most AA's in Southwest Philadelphia speak English and Spanish. This assessment will give an idea of the target population's 
views, knowledge, culture, environmental barriers, reason for the lack of access to healthcare and their attitudes concerning the issue. The questionnaires and the survey will be distributed in places such as community churches, markets, shops and community parks, which are frequently visited by the AA's. The questions in will be structured in such a way that it will not offend the AA's and will be easy for them to answer. There will be two types of questions, one will be yes or no and the other type will be a multiple choice. The AA's will be provided with a giveaway incentive for completing the questionnaire. Some of the questions are:

1. Would you say that in general your health is_(Excellent/ Very good/ Good/ Fair/ Poor/ Do not know)

2. Are you aware of the health condition called hypertension/ increased blood pressure? (Yes/No)

3. Do you know the effects of hypertension on the organs of the body such as heart, kidney? (Yes/No)

4. Do you feel regular blood pressure screening is important after the age of 35-40years? (Yes/No)

5. Do you have any kind of health insurance or health coverage? (Yes/No). Check all that apply. (Medicaid / Medicare/Aetna/ Private health insurance/Other, please specify)

6. Was there a time in the past six months when you needed to visit a doctor but couldn't because of the cost? (Yes/No/Not sure/Not interested in answering)

7. During the past month did you participate in any physical activities such as running, jogging, gardening, workouts? (Yes/No/Don't know)

8. About how long it has been since you last visited a doctor for a routine health checkup or routine blood pressure screenings? (One month/Six months/One year/More than a year)

9. Is there any specific reason for not undergoing regular blood pressure screening? (Lack of sufficient income/Lack of time/ Lack of access/Lack of transportation/Lack of knowledge and awareness)

10. What does your diet usually consists of? Check all that apply. (Pizza/Burger/Fried items/Vegetable/Salads/Fruits/Cereal/Other, please specify in detail)

The phase two of the PPM is the epidemiological assessment phase which provides knowledge about the factors that might influence the outcome of the intervention. Low socio-economic status, racial and ethnic disparities, nutrition, geographic factors and medical factors are the some of the causes of hypertension in AA's. ${ }^{6}$ Data from various sources can be obtained to determine the influence of various factors on hypertension. The data from National Health Statistics Report and USA.gov will provide the severity of the issue on the National level. Data about the race, ethnicity and income will be helpful in determining the influence of these factors on the issue. In addition to this, data from various local health organizations, Philadelphia Department of Public Health can be used to determine the prevalence of the issue and its causal factors in Southwest Philadelphia. Data from Centers for Disease Control and Prevention (CDC), AHA and NHLBI will provide the statistics and the research evidence associated with the predisposing factors and the medical aspect of hypertension

\section{in AA's (Table 1)}

The behavioral factors associated with hypertension in AA's involve regular BP screening after the age of 40years. Hypertension is asymptomatic in early stages, so regular screening will help determine the blood pressure levels. The lifestyle factors include unhealthy dietary habits such as consumption of fatty foods and sodium enriched foods, lack of physical activities and low socio-economic status. The environmental factors include the neighborhoods in which AA's live, access to health care and access to healthy foods. In phase one and phase two of the PPM, the objectives of the intervention are created. ${ }^{4}$ The phase three of the PPM deals with examining the behavior, lifestyle and the environmental factors associated with the issue. In this phase, you determine how the factors can be modified to solve this issue. The predisposing factors include lack of access to health care due to various reasons such as low income, lack of transportation, lack of awareness about hypertension and the risks associated with untreated hypertension. If they learn about the risk of untreated hypertension, they might consider taking care of their blood pressure level. Low socio-economic status leading to stress and improper dietary habits are also predisposing factors associated with this issue. ${ }^{7}$ A Reinforcing factor includes motivation from family members and members of the religious organization to undergo screening which will lead to health promotion. Including faith-based approaches has encouraged the AA's to control their hypertension. ${ }^{8}$

The phase four of the PPM deals with identifying best practices and model approaches relevant to the proposed intervention. The organizational, administrative and the regulatory policies are the vital components for the success of an intervention. The SHAPE-IT program which is the Stroke, Hypertension and Prostrate Education Intervention Team by Philadelphia Department of Public Health, Health Promotion Council of Southeastern Pennsylvania, Office of Advance Population Health (TJU), the Division of Genetics and Preventive Medicine of the Department of Medicine (TJU) and the Department of Family and Community Medicine of Thomas Jefferson University (TJU) is the gold standard for the proposed intervention. The reason for choosing this intervention is because its target is to control hypertension among AA's by encouraging them to undergo regular screening. Another program which forms the basis of this intervention is the FAITH program that includes faithbased approaches in treating hypertension among the AA's with the help of church members. ${ }^{8}$ The primary health care centers and the community-based organizations located in Southwest Philadelphia should be included in the intervention.

\section{Target audience \& rationale for focus}

The proposed intervention's target population is the low-income AA's over the age of 40years who have hypertension as well as who are at risk of having hypertension living in Southwest Philadelphia. About 67million people in United States are affected by hypertension. ${ }^{9}$ The main reason for choosing AA's as the target population is that AA's are at increased risk of being affected by hypertension as compared to other racial and ethnic groups. ${ }^{10}$ Hypertension is more common in AA's adults over the age of 40years. ${ }^{11}$ Hence, hypertension which is a growing public health concern is being targeted. About $44.3 \%$ of the total population in Philadelphia is AA's. ${ }^{2}$ Philadelphia has the highest number of AA's who are below the poverty line. ${ }^{13}$ Most of the population in Southwest Philadelphia are below the poverty line and thus has been chosen as the focus area. 


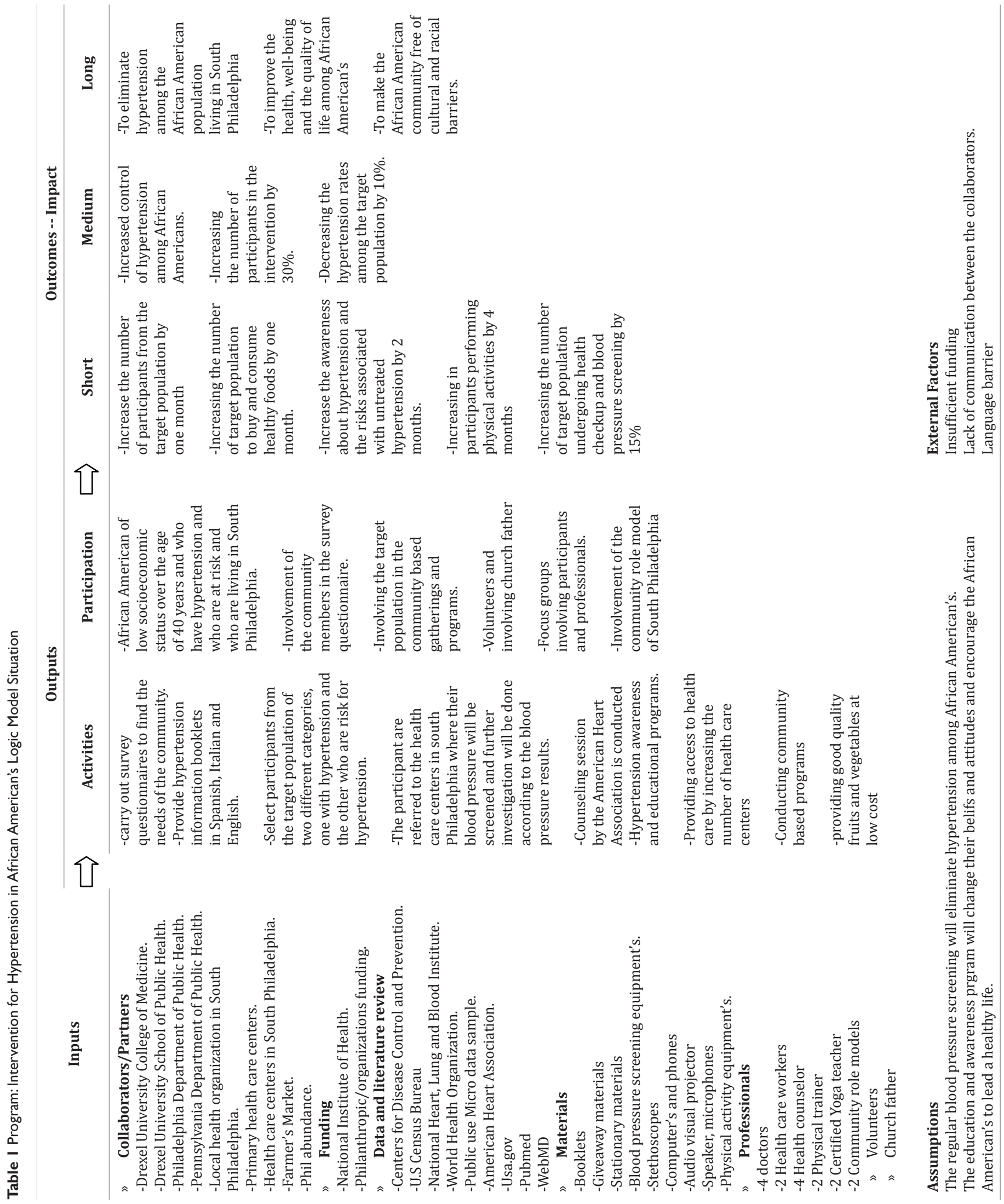


The AHA, primary health care centers, hospitals in Southwest Philadelphia, community-based organizations and community leaders are the key stakeholders in this intervention. Proper coordination and communication between the stakeholders will help achieve success in the intervention. The primary health care center is the place where the blood pressure screening and other medical aspects of the intervention will be carried out. The AHA will carry out various activities and events to encourage the AA's to control hypertension. The community organizations will organize lectures and give out information booklets. The community leaders will give motivational speeches to the target population. Participation by the family members of AA, community members and head of the community organization of Southwest Philadelphia, will promote the public to participate in the intervention that lead to successful intervention. Members of the religious centers are also stakeholders in this intervention as faith-based motivation works well on this issue.

\section{Project description}

The intervention will focus on the low-income AA's above the age of 40 living in Southwest Philadelphia, who has hypertension as well as who is at risk of getting hypertension. The funding for the intervention will be obtained from the National Institute of health The intervention will primarily take place at the health care centers located in Southwest Philadelphia. A healthcare worker will be appointed to select the appropriate participants for the intervention. The health care worker will also place participants in categories, one who already have hypertension and the other who are at risk of having hypertension. Four heart specialists from the AHA will be appointed as physician at the health care centers for the intervention. Two of the physician's will examine the participants with hypertension, do various health tests specific to each individual's clinical findings and prescribe medicines. The other two physicians will examine the population at risk, by performing BP screening and guiding them on the basis of their test results. The participants will then be referred to AHA where various events will be conducted to promote the AA's to control BP. The AHA will counsel the AA's regarding the importance of healthy foods and physical activities and will provide giveaways at the end of the session.

The community organization will conduct sessions which will include giving out information booklets about hypertension in Spanish and English language. Various sessions will be conducted that will include physical activity demonstrations, yoga exercises, healthy cooking classes and interactive gaming session which provide knowledge for their information. The community leaders of Southwest Philadelphia will give motivational speeches so as to encourage AA's to control their hypertension. Religious discourse in the churches of Southwest Philadelphia by church fathers will be conducted weekly and speeches about the importance of health will be inculcated in the discourse. It will focus on the racial and cultural factors and help the AA's resolve the barriers associated with it. These steps in the intervention will create awareness among the AA's about hypertension control. Organizing farmer's market one alternate day is also a part of the intervention. The farmer's market will provide good quality fruits and vegetables at low cost which is affordable to the low-income AA (Table 1).

\section{Evaluation}

The smart evaluation consists of the phase six and phase seven which are the process evaluation phase and the impact evaluation phase respectively. The process evaluation phase deals with proper assessment of the intervention procedure. It also determines whether the target population is properly reached in this intervention.
Evaluation should be done to assess whether the physician's timing and the participant's appointment at the healthcare centers match by checking the hospital records. Assessment will also include determining whether the participants are attending the counseling session at the AHA by keeping a count of their participation. Are the sessions taking place according to the convenience of the participants by interviewing the participants, are the giveaways given to all the participants who attend the session by keeping a list of giveaways provided to each participant. The phase seven is the impact evaluation phase that deals with determining the impact of the intervention on AA's. To make sure the intervention is on the right path, it essential to assess whether the intervention is having the positive impact on the population. This phase will help in assessing the initial success of the intervention. Two surveys will be conducted to evaluate the impact of the intervention on AA's, one will be after one month of the intervention and the other will be after six months of the intervention. Interviews would be conducted to ask the participants whether they are satisfied with the intervention outcomes. Community meetings will be conducted to ask about the participants view about the impact of the intervention on their lives.

The phase eight is the outcome evaluation phase which focuses on the final output of the intervention. The main outcome of the intervention is to control and prevent hypertension among AA's and to promote a healthy life. This phase will help determine the success rate of the intervention. In this phase, we determine whether the intervention is really providing the outcome, the way it was needed by the community. A blood pressure screening after the intervention period will determine whether hypertension rate has been reduced. A survey will be conducted after the intervention, which will help to determine the understanding and the knowledge gained by the target population. The assets of the intervention play an important role in helping the intervention to reach its goal of improving the quality of life for AA's by controlling and preventing hypertension. The community-based approach to the intervention is one of the assets, as it is more effective in reaching the minds of the AA's. Participants who participate in the sessions will be given giveaways which constitutes as one of the assets. Religious and faith-based approach also bolster the intervention by encouraging the AA's to live a healthy life. The barriers of the intervention will be improper communication between either physician and the participants or counselors and participants. This problem can be overcome by appointing translators. The funds obtained are not sufficient for the intervention. Donations could be obtained from various philanthropies to aid in funding for the intervention. The intervention only focuses on the hypertension aspect of healthcare. It should focus on the overall health and wellbeing of AA.

\section{Acknowledgements}

None.

\section{Conflict of interest}

The author declares no conflict of interest.

\section{References}

1. World Health Organization (WHO). Cardiovascular diseases (CVDs). Fact sheet $\mathrm{N}^{\circ} 317$. Geneva: World Health Organization; 2015.

2. Riaz K, Ahmed A, Ali YS. Hypertensive Heart Disease. USA: Medscape; 2013.

3. Philadelphia Department of Public Health (PDPH). Community Health Assessment. USA: Philadelphia Department of Public Health; 2014. p. $1-132$. 
4. Green LW, Rabinowitz P. Community Tool Part A, Chapter 2, Section 2 PRECEDE/PROCEED. USA: KUWG Publisher; 2012.

5. National Heart Lung and Blood Institute. Who is at risk for blood pressure? USA: National Institutes of Health; 2012.

6. American Heart Association. Risk Factors. Statistical Fact Sheet. USA American Heart Association; 2014.

7. Xanthos C, Henrie M, Treadwell, et al. Social determinants of health among African-American men. WPMH GmbH. 2010;7(1):11-19.

8. Lancaster KJ, Schoenthaler AM, Midberry SA, et al. Rationale and design of Faith-based Approaches in the Treatment of Hypertension (FAITH), a lifestyle intervention targeting blood pressure control among black church members. Am Heart J. 2014;167(3):301-307.
9. Centers for Disease Control and Prevention (CDC). High Blood Pressure Sheet. USA: Division for Heart Disease and Stroke Prevention, National Center for Chronic Disease Prevention and Health Promotion; 2014.

10. Beckerman J. Hypertension/High Blood Pressure Health Center. USA: WebMD; 2013.

11. Schiller JS, Lucas JW, Peregoy JA. Summary Health Statistics for U.S Adults: National Health Interview Survey, 2011. National Center for Health Statistics. Vital Health Stat. 2012;10(256).

12. US Census Bureau. Population Estimates. USA: QuickFacts Beta, United States Census Bureau; 2014.

13. Rabinowitz P. Community Tool Box, Chapter 4, Section 3: Gaining Public Support for Addressing Community Health and Development Issues. USA: KUWG Publisher; 2013. 